Original Article

\title{
Comparative assessment of knee extensor and flexor muscle strength measured using a hand-held vs. isokinetic dynamometer
}

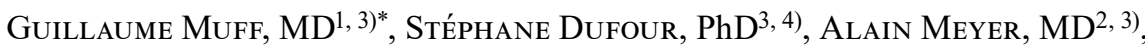
François Severac, MD ${ }^{3)}$, Fabrice Favret, PhD 3 , 4), Bernard Geny, MD, $\mathrm{PhD}^{2,3)}$, Jehan LecocQ, MD, $\mathrm{PhD}^{1,3)}$, Marie-Eve Isner-Horobeti, MD, $\mathrm{PhD}^{1,3)}$

1) Department of Physical Medicine and Rehabilitation, University Institute of Rehabilitation Clemenceau, Strasbourg University: 45 boulevard Clemenceau, Strasbourg 67082, France

2) Institute of Physiology, Faculty of Medicine and University Hospital, Strasbourg University, France

3) Federation of Translational Medicine (FMTS), Strasbourg University, France

4) Faculty of Sports Sciences, Strasbourg University, France

\begin{abstract}
Purpose] To compare measurements of knee extensor and flexor muscle strength performed using a hand-held dynamometer and an isokinetic dynamometer in apparently healthy subjects. [Subjects and Methods] Thirty adult volunteers underwent knee muscle strength evaluation using an isokinetic or a hand-held dynamometer. [Results] Strong positive correlations were found between the 2 methods, with correlation coefficients $\mathrm{r}$ ranging from 0.72 (95\% confidence interval [CI], 0.48-0.86) to 0.87 (95\% CI, 0.75-0.94), depending on the muscle group and the isokinetic evaluation mode. The reproducibility of the hand-held dynamometer findings was good, judged by a coefficient of variation of $3.2-4.2 \%$. However, the correlation between the 2 methods for the assessment of flexor/extensor ratios ranged from -0.04 to 0.46 . [Conclusion] Knee extensor and flexor muscle strength recorded with a hand-held dynamometer is reproducible and significantly correlated with the isokinetic values, indicating that this method may in some cases be a useful replacement for isokinetic strength measurement. However, for strength ratio assessment, and when judged against the isokinetic standard, a hand-held dynamometer is not a valid option.

Key words: Muscle, Strength, Dynamometer
\end{abstract}

(This article was submitted Mar. 8, 2016, and was accepted May 23, 2016)

\section{INTRODUCTION}

Numerous pathological conditions can lead to alterations in muscle performance and locomotor ability ${ }^{1-7)}$. The strength of the knee extensor and flexor muscles has been extensively studied and is a relevant clinical indicator of health status and functional capacity in coronary artery disease ${ }^{8)}$, neuromuscular diseases ${ }^{9)}$, the elderly ${ }^{10)}$, renal disease ${ }^{11)}$, and fibromyalgia ${ }^{12)}$. In sports medicine, knee extensor and flexor muscle strength is often monitored with the aim of preventing or treating orthopedic as well as muscular injuries ${ }^{13-19)}$. Therefore, practitioners need to have reliable tools for easy and reproducible assessment of muscle strength. The isokinetic concept of exercise ${ }^{20)}$ is currently considered the best method for the assessment of knee extensor and flexor strength because of its well-established validity and reproducibility ${ }^{21-25)}$. However, the acquisition and maintenance costs, the time required to perform an isokinetic assessment, as well as the absence of portability of isokinetic dynamometers can limit their use. Conversely, hand-held dynamometers (HHD) are characterized by specific features such as low cost, portable design, and rapid data acquisition. These systems are increasingly used, both in the clinic

*Corresponding author. Guillaume Muff (E-mail: guillaume.muff@gmail.com)

(C2016 The Society of Physical Therapy Science. Published by IPEC Inc.

This is an open-access article distributed under the terms of the Creative Commons Attribution Non-Commercial No Derivatives (by-nc-nd) License $<$ http://creativecommons.org/licenses/by-nc-nd/4.0/>. 
and in research. An HHD provides reliable and reproducible results when used for the evaluation of muscle groups that produce little or moderate amounts of force. However, the results obtained for strong muscles, such as the knee extensors, are less convincing ${ }^{26-28)}$. A systematic review comparing muscle strength assessment by HHD and isokinetic testing ${ }^{29)}$ showed a positive correlation between these methods. However, the heterogeneity of the protocols and devices evaluated made the interpretation of the results difficult. Therefore, the aim of this study was to: 1) assess the validity of HHD vs. isokinetic dynamometry in the evaluation of knee extensor and flexor muscle strength, 2) establish the reproducibility of HHD measurements of knee extensor and flexor muscle strength, and 3) compare the flexor/extensor ratios obtained with the 2 methods.

\section{SUBJECTS AND METHODS}

The study was carried out at the Muscle Evaluation Unit at the Clemenceau Rehabilitation University Institute (Strasbourg, France) and involved 30 healthy volunteers (Table 1).

Subjects were recruited by means of advertisement in the university network from January 2015 to November 2015. Inclusion criteria were as follows: age older than 18 years, and without cardiovascular disorders, osteoarticular diseases of the knees, or previous knee osteoligamentous or thigh muscle injuries.

The study protocol was approved by the local ethics committee. All participants were fully informed about the risks and procedures associated with the experiment and provided written informed consent to participate in the study. The trial registration number on ClinicalTrials.gov is ID: NCT02566122.

Each participant completed a 10-min warm-up on a cycle ergometer (power: $1 \mathrm{~W} / \mathrm{kg}$ body weight; pedaling rate: 70-80 rpm). The testing method sequence (HHD vs. isokinetic testing) was established according to a randomization table prepared by the Biostatistics Department of the Strasbourg University Hospitals using R software version 3.2.2 (R Core Team (2015). R Foundation for Statistical Computing, Vienna, Austria. URL https://www.R-project.org/). The randomization of the subjects was generated by a draw using a Bernoulli distribution with a parameter of 0.5 to maintain a mean balance in the test order. The randomization was simple, without any blocking variable. A recovery period of 20 min was implemented between testing methods. For both methods, muscle strength was first measured in the dominant leg, and after a 10-min rest, in the non-dominant leg. To avoid inter-examiner variations, all measurements were carried out by a single investigator, specialized in Physical Medicine and Rehabilitation (male, 31 years old, $180 \mathrm{~cm}$, and $90 \mathrm{~kg}$ ).

The strength of the extensor and flexor muscles of both knees was assessed using an isokinetic dynamometer (Con-Trex MJ; CMV AG, Dübendorf, Switzerland). Each participant was positioned on an adjustable chair with the back set at $90^{\circ}$ of posterior inclination. The sitting position was set according to the manufacturer's recommendations. The knee range of motion was set at $90^{\circ}$ (from $100^{\circ}$ to $10^{\circ}$ of flexion; $0^{\circ}$ corresponding to the complete extension of the knee) after having aligned the leg segment to anatomical zero. Finally, a measurement of gravity for the leg segment to be tested was carried out over the entire range of motion. The protocol of isokinetic evaluation included 6 series of contractions on the dominant and nondominant sides including concentric, isometric, and eccentric evaluations of the knee extensor and flexor muscles (Table 2). A recovery period of $60 \mathrm{~s}$ was observed between each maximal series. Before each maximal series, participants carried out a «trial» submaximal series to become familiar with the contraction mode and the angular speed. Participants did not have any visual feedback of their performance. Before the test, participants received standardized oral information on the experimental procedure and were verbally encouraged in a standardized manner by the operator during the test. Following 10 min of rest after the evaluation of the dominant leg, muscle testing was performed in exactly the same way for the non-dominant side.

The strength of the extensor and flexor muscles of both knees was assessed with hand-held dynamometry (MicroFET2 ${ }^{\circledR}$, Hogan Health Industries, Inc., UT, USA). This dynamometer is a portable digital instrument than can be held in the palm of the hand giving muscle strength measurements in kilogram-force (kgf). As with manual muscle testing, this dynamometer is placed between the leg segment to be evaluated and the examiner's hand. Participants were asked to sit with their legs dangling over the end of a standard, adjustable examination table, with hips and knees flexed to $90^{\circ}$, in order to have a distance of $1-2 \mathrm{~cm}$ between the popliteal fossae and the table end. The height of the examination table was adjusted to have a distance of about $10 \mathrm{~cm}$ between the participant's feet and the floor. Participants had to hold the side-edges of the table with their hands and carry out a maximal isometric voluntary contraction for $5 \mathrm{~s}$. The examiner positioned a knee on the floor, with the arm fully extended in front of the lower limb to be tested. To evaluate the knee extensor muscles, the dynamometer was placed on the anterior part of the lower leg, above the talotibial joint line (Fig. 1.A). The examiner placed one of his feet against the wall to better resist muscle contraction. To evaluate the knee flexor muscles, the dynamometer was placed on the posterior part of the leg, 1-2 cm above the lateral malleolus (Fig. 1.B). In both cases, the examiner produced a resistance force in the horizontal direction to counter the force developed by the participant and maintain an isometric contraction of the knee extensor and flexor muscles. The evaluation started with the extensor muscles of the dominant leg, followed by the flexors of the dominant side. After a recovery period of $10 \mathrm{~min}$, the knee extensor and flexor muscles of the non-dominant leg were assessed using the same experimental design. For each muscle group, participants carried out 3 isometric maximal voluntary contractions of $5 \mathrm{~s}$. A 60-s recovery period was observed between each muscle contraction. The position of the leg to be tested was verified initially with the help of a standard goniometer. Before the evaluation, participants received standardized oral information on the test procedure and were encouraged orally during the test.

For each isokinetic series, the measurement criterion was the peak torque (PT) obtained during each series, expressed in 
Table 1. Characteristics of the population $(n=30)$

\begin{tabular}{lcc}
\hline & Mean \pm SD & Range \\
\hline Age $($ years $)$ & $32.8 \pm 11.5$ & $20-59$ \\
Height $(\mathrm{cm})$ & $176.9 \pm 10.3$ & $160-197$ \\
Weight $(\mathrm{kg})$ & $74.5 \pm 15.8$ & $52-109$ \\
Gender & Males $(\mathrm{n}=20)$ & Females $(\mathrm{n}=10)$ \\
Laterality & Right $(\mathrm{n}=27)$ & Left $(\mathrm{n}=3)$ \\
Test sequence & ISO then HHD $(\mathrm{n}=16)$ & HHD then ISO $(\mathrm{n}=14)$ \\
\hline
\end{tabular}

ISO: isokinetic dynamometer; HHD: hand-held dynamometer
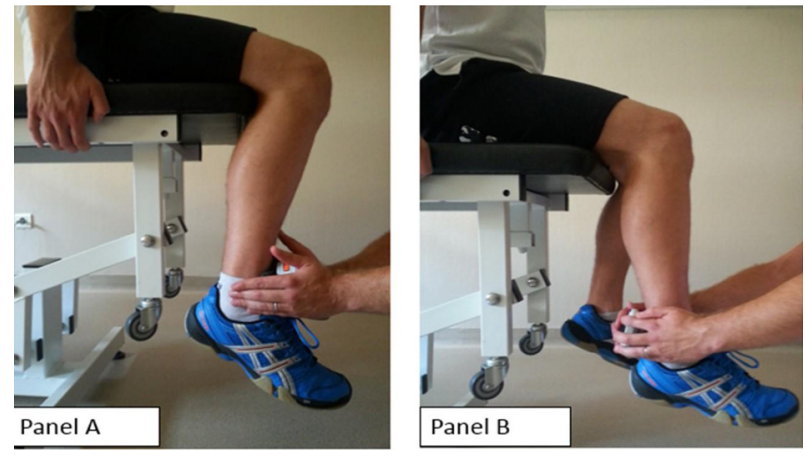

Fig. 1. Position of an individual subject for the evaluation of knee-extensor (panel A) and Knee-flexor (panel B) muscles using the hand-held dynamometer

Table 2. Protocol for muscle strength measurement with the isokinetic dynamometer

\begin{tabular}{lccccc}
\hline Series & Speed & Muscles & Mode & Repetitions & ROM/position \\
\hline Warm-up & $180 \%$ sec & Ext/Flex & Concentric & 10 & $90^{\circ}$ \\
Warm-up & $120 \% / \mathrm{sec}$ & Ext/Flex & Concentric & 10 & $90^{\circ}$ \\
Series 1 & $60 \% \mathrm{sec}$ & Ext/Flex & Concentric & 3 & $90^{\circ}$ \\
Series 2 & $180 \% \mathrm{sec}$ & Ext/Flex & Concentric & 6 & $90^{\circ}$ \\
Series 3 & $0 \% / \mathrm{sec}$ & Ext & Isometric & 1 & $90^{\circ}$ of flexion \\
Series 4 & $0 \% / \mathrm{sec}$ & Flex & Isometric & 1 & $90^{\circ}$ of flexion \\
Series 5 & $60 \% \mathrm{sec}$ & Ext & Eccentric & 3 & $90^{\circ}$ \\
Series 6 & $60 \% \mathrm{sec}$ & Flex & Eccentric & 3 & $90^{\circ}$ \\
\hline
\end{tabular}

Ext: knee extensors; Flex: knee flexors; ROM: range of motion

Newton-meters $(\mathrm{Nm})$.

The maximal force developed during each contraction, expressed in kilogram-force (kgf), was recorded. For each muscle group, the maximal force was averaged over the 3 isometric contractions and used as the measurement criterion.

Thus, the 2 obtained variables were the peak torque $(\mathrm{Nm})$ and the maximal force (kgf).

By assuming a correlation of 0.6 between the 2 measurements and a total magnitude of the confidence interval of 0.45 around the estimate, a sample size of 28 subjects was needed. The reproducibility of the HHD measurement was established using the coefficient of variation over 3 consecutive measures. According to Stokes, reproducibility was considered acceptable when the coefficient of variation was $15 \%$ or below ${ }^{30}$. The statistical analysis was performed independently by the Biostatistics Department of the Strasbourg University Hospitals. All analyses were carried out using R version 3.2.2, with all the required packages, in the most updated version available at the time of the data analysis. The Gaussian character of the variables was assessed with the Shapiro-Wilk test. When 2 variables followed a normal distribution, the Pearson correlation coefficient was used; if not, the Spearman correlation coefficient was employed. Concerning the correlations, the «test sequence» and the «laterality» effects were evaluated by ensuring that the confidence intervals (CI) at $95 \%$ for each variable were overlapping in the 2 modalities. Values are presented as mean \pm SD unless otherwise indicated.

\section{RESULTS}

No «test sequence» or «laterality» effect was found. The results obtained with the 2 evaluation modes on the whole population are summarized in Table 3. The strengths measured for the knee extensor muscles were greater than the corresponding values for the knee flexor muscles, whatever the mode and velocity of the muscle actions $(\mathrm{p}<0.05)$.

The knee flexor/extensor strength ratios did not change according to the evaluation mode, with no difference between the dominant and non-dominant sides (Table 4).

The knee extensor muscle strength was significantly greater than for the knee flexor muscles, both on the dominant and non-dominant side $(\mathrm{p}<0.05)$. There was no difference between the dominant and non-dominant side in a given muscle group (Table 3). The ratios of knee flexor/extensor muscle strength were similar in dominant and non-dominant sides and were not significantly different compared to isokinetic ratios (Table 4).

The coefficient of variation of the 3 isometric muscle strength measurements using the microFET 2 HHD ranged from 
Table 3. Results of the muscle strength measurements

\begin{tabular}{|c|c|c|c|}
\hline & $\begin{array}{c}\text { Mean } \pm \text { SD } \\
\text { Ecart-type }\end{array}$ & Range & Median \\
\hline \multicolumn{4}{|c|}{ Isokinetic (Nm) } \\
\hline \multicolumn{4}{|c|}{ Concentric $60 \% / \mathrm{sec}$} \\
\hline EXT D & $179.1 \pm 52.2 * \$ \#$ & $100.3-275.8$ & 180.7 \\
\hline EXT ND & $165.4 \pm 52.0^{*}$ & $90.5-260.3$ & 155.3 \\
\hline FLEX D & $91.9 \pm 28.5 \$$ & $51.1-164.7$ & 93.8 \\
\hline FLEX ND & $85.1 \pm 25.5$ & $51.0-143.8$ & 78.1 \\
\hline \multicolumn{4}{|c|}{ Concentric $180^{\circ} / \mathrm{sec}$} \\
\hline EXT D & $132.3 \pm 39.4 * \$$ & $75.4-207.8$ & 136.8 \\
\hline EXT ND & $130.4 \pm 39.6^{*}$ & $71.5-204.2$ & 134.1 \\
\hline FLEX D & $72.2 \pm 22.9 \$$ & $32.1-115.3$ & 72.8 \\
\hline FLEX ND & $71.8 \pm 24.1 \$$ & $32.0-141.6$ & 69.2 \\
\hline \multicolumn{4}{|c|}{ Isometric $0 \%$ sec } \\
\hline EXT D & $177.2 \pm 57.3^{*}$ & $89.5-291.8$ & 181.2 \\
\hline EXT ND & $167.4 \pm 56.6^{*}$ & $88.0-281.0$ & 162.2 \\
\hline FLEX D & $84.0 \pm 25.2$ & $48.0-153.7$ & 84.7 \\
\hline FLEX ND & $79.3 \pm 25.9$ & $45.0-144.2$ & 73.1 \\
\hline \multicolumn{4}{|c|}{ Eccentric $60 \% \mathrm{sec}$} \\
\hline EXT D & $201.9 \pm 61.9^{*}$ & $110.9-357.5$ & 191.5 \\
\hline EXT ND & $195.5 \pm 69.8^{*}$ & $111.8-357.0$ & 169.9 \\
\hline FLEX D & $123.1 \pm 44.1$ & $69.5-252.3$ & 111.6 \\
\hline FLEX ND & $116.7 \pm 36.8$ & $65.9-210.3$ & 103.8 \\
\hline \multicolumn{4}{|l|}{ HHD (kgf) } \\
\hline EXT D & $52.9 \pm 16.7 *$ & $22.7-87.4$ & 57.6 \\
\hline EXT ND & $51.1 \pm 17.2^{*}$ & $22.2-84.3$ & 51.8 \\
\hline FLEX D & $26.7 \pm 6.0$ & $17.4-40.9$ & 27.2 \\
\hline FLEX ND & $26.9 \pm 5.3$ & $18.2-38.9$ & 27.1 \\
\hline
\end{tabular}

EXT: knee extensors; FLEX: knee flexors; D: dominant side; ND: non-dominant side; SD: standard deviation. ${ }^{*} \mathrm{p}<0.05$ in EXT vs. FLEX in similar conditions. $\$ \mathrm{p}<0.05$ vs. Eccentric $60 \% \mathrm{~s}$ in similar muscles. $\# \mathrm{p}<0.05$ Concentric $60 \%$ s vs. concentric $180 \%$ in similar muscles
Table 4. Flexor/Extensor ratios in isokinetic and hand-held dynamometry

\begin{tabular}{lcc}
\hline & Dominant Side & $\begin{array}{c}\text { Non dominant } \\
\text { Side }\end{array}$ \\
\hline Concentric $60 \% \mathrm{sec}$ & $52 \pm 7.0 \%$ & $53 \pm 9.7 \%$ \\
Concentric $180 \% \mathrm{sec}$ & $55 \pm 7.0 \%$ & $55 \pm 8.4 \%$ \\
Isometric $0 \% \mathrm{sec}$ & $49 \pm 8.7 \%$ & $49 \pm 11.0 \%$ \\
Eccentric $60 \% \mathrm{sec}$ & $61 \pm 8.1 \%$ & $61 \pm 10.9 \%$ \\
HHD & $53 \pm 12.9 \%$ & $57 \pm 14.6 \%$ \\
\hline
\end{tabular}

Data are means \pm SD. HHD: hand-held dynamometer

3.2 to $4.2 \%$ according to the muscle group evaluated. Mean difference (absolute and relative values) as well as limits of agreement were similar between muscle groups (Table 5).

The correlation coefficient between the isokinetic and HHD muscle strength values ranged from $\mathrm{r}=0.87(0.75-0.94)$ to $\mathrm{r}=0.72(0.48-0.86)(\mathrm{p}<0.01)$, indicating that the correlation between testing methods was generally good (Table 6).

The flexor/extensor ratios were significantly correlated between the HHD and isokinetic dynamometer in concentric $180^{\circ}$ / sec and isometric $0 \%$ sec modes on the dominant and non-dominant sides $(\mathrm{p}<0.05)$. The flexor/extensor ratio measured in concentric $60^{\circ} / \mathrm{sec}$ mode was correlated to the HHD only for the non-dominant side. No correlation was observed for the flexor/extensor ratios between HHD and isokinetic eccentric $-60 \%$ sec mode (Table 7).

\section{DISCUSSION}

The results of the present study show: 1) good reproducibility of the HHD for knee extensor/flexor muscle strength assessment, 2) clinically acceptable agreement between the values obtained with the HHD versus the isokinetic dynamometer, and 3 ) the need for caution when interpreting the flexor/extensor ratios using HHD compared to isokinetic dynamometry.

The muscle strength values using isokinetic dynamometry recorded in this study are in good agreement with those found in the literature ${ }^{31)}$, particularly in the study by Maffiuleti et al. in $2007^{23)}$. For a similar population of healthy individuals (age: $30 \pm 5$ vs. $33 \pm 11$ years old; height: $175 \pm 8$ vs. $177 \pm 10 \mathrm{~cm}$; weight: $70 \pm 13 \mathrm{vs.} 74 \pm 16 \mathrm{~kg}$ ), the strength measurements using the Con-Trex isokinetic module were very close, with a peak torque for the knee extensors on the dominant side of 178 \pm 46 vs. $179 \pm 52 \mathrm{Nm}$ (concentric at $60^{\circ} / \mathrm{s}$ ); $132 \pm 38$ vs. $132 \pm 39 \mathrm{Nm}$ (concentric $180^{\circ} / \mathrm{s}$ ), and $202 \pm 68$ vs. $202 \pm 62 \mathrm{Nm}$ (eccentric $60^{\circ} / \mathrm{s}$ ) when compared with our values. 
Table 5. Reproducibility of muscle strength evaluation with microFET 2 hand-held dynamometer

\begin{tabular}{lccccc}
\hline & $\begin{array}{c}\text { Coefficient } \\
\text { of variation }\end{array}$ & \multicolumn{2}{c}{ Mean Difference } & \multicolumn{2}{c}{ Limits of agreement } \\
\cline { 2 - 7 } & $(\%)$ & $(\mathrm{kgf})$ & $(\%)$ & $(\mathrm{kgf})$ & $(\%)$ \\
\hline Dominant Knee Extensors & 3.2 & $0.1 \pm 3.7$ & $0.1 \pm 7.0$ & $-7.3-7.6$ & $-13.8-14.0$ \\
Non-dominant Knee Extensors & 3.3 & $0.0 \pm 3.7$ & $0.4 \pm 7.4$ & $-7.5-7.5$ & $-15.1-14.4$ \\
Dominant Knee Flexors & 4.2 & $0.4 \pm 2.5$ & $1.4 \pm 8.9$ & $-4.6-5.4$ & $-16.4-19.2$ \\
Non-dominant Knee Flexors & 3.7 & $0.3 \pm 2.1$ & $0.9 \pm 7.7$ & $-3.9-4.6$ & $-14.4-16.3$ \\
\hline
\end{tabular}

Table 6. Correlation coefficients and $95 \%$ confidence intervals for knee muscle strength assessments obtained by hand-held (HHD) and isokinetic dynamometers (Con-Trex)

\begin{tabular}{lcccc}
\hline & \multicolumn{4}{c}{ Con-Trex } \\
\cline { 2 - 5 } & Eccentric & Isometric & Concentric & Concentric \\
\cline { 2 - 5 } & $60 \% \mathrm{sec}$ & $0 \% \mathrm{sec}$ & $60 \% \mathrm{sec}$ & $180 \% \mathrm{sec}$ \\
\hline HHD & & & \\
Extensors D & $\mathrm{r}=0.87$ & $\mathrm{r}=0.85$ & $\mathrm{r}=0.87$ & $\mathrm{r}=0.85$ \\
& $(0.75-0.94)$ & $(0.70-0.92)$ & $(0.73-0.93)$ & $(0.71-0.93)$ \\
Extensors ND & $\mathrm{r}=0.84^{*}$ & $\mathrm{r}=0.87$ & $\mathrm{r}=0.86$ & $\mathrm{r}=0.87$ \\
& $(0.69-0.92)$ & $(0.74-0.94)$ & $(0.72-0.93)$ & $(0.74-0.94)$ \\
Flexors D & $\mathrm{r}=0.80^{*}$ & $\mathrm{r}=0.75$ & $\mathrm{r}=0.81$ & $\mathrm{r}=0.85$ \\
& $(0.62-0.90)$ & $(0.53-0.87)$ & $(0.63-0.91)$ & $(0.70-0.93)$ \\
Flexors ND & $\mathrm{r}=0.75^{*}$ & $\mathrm{r}=0.72^{*}$ & $\mathrm{r}=0.83^{*}$ & $\mathrm{r}=0.82$ \\
& $(0.53-0.87)$ & $(0.48-0.86)$ & $(0.67-0.92)$ & $(0.66-0.91)$ \\
\hline
\end{tabular}

*Spearman correlation coefficient; D: dominant side; ND: non-dominant side. All coefficients of correlation are significant $(\mathrm{p}<0.01)$.

Table 7. Coefficients of correlation for knee flexor/extensor muscle strength ratios obtained with hand-held or isokinetic dynamometry

\begin{tabular}{lcc}
\hline & Dominant & Non dominant \\
\hline Concentric $60^{\circ} / \mathrm{sec}$ & 0.23 & $0.46 *$ \\
Concentric $180^{\circ} / \mathrm{sec}$ & $0.39 *$ & $0.45^{*}$ \\
Isometric $0 \% \mathrm{sec}$ & $0.37 *$ & $0.46 *$ \\
Eccentric $60 \% \mathrm{sec}$ & -0.04 & 0.13 \\
\hline${ }^{*} \mathrm{p}<0.05$ & &
\end{tabular}

Similarly, the muscle strength values recorded with the HHD are in agreement, albeit with greater absolute data, with literature data ${ }^{32,33)}$ and with the recent study by Douma et al., who established reference values for knee extensor and flexor muscle strength in the active Dutch population by using the MicroFet $2 \mathrm{HHD}^{34)}$. The magnitude of difference between our values and previously published data using HHD is likely related to the characteristics of the participants, who were younger and fitter in our study compared to previous studies.

Greater limits of agreement were previously observed for reproducibility of muscle strength using HHD in strong muscle groups (i.e., knee extensors) compared to weaker muscle groups (i.e., knee or elbow flexors) ${ }^{34)}$. Consequently, HHD was not considered as suitable methodology for muscle strength assessment in knee extensors ${ }^{35}$. Conversely, our results are at odds with this view as we report that the reproducibility of muscle strength assessment is not altered by the level of muscle strength developed by the subjects. Indeed, the variation coefficients were lower for the 3 measures of knee extensors compared to knee flexors, despite higher levels of absolute strength in the former. Similarly, we did not observe greater limits of agreement (LOA) in the knee extensors compared to the knee flexors. Of note, absolute values of LOA in the present study are somewhat lower than those previously published ${ }^{34}$. We suggest that if HHD devices are manipulated by experienced operators, HHD can be used for the evaluation of muscle strength, even in strong muscles such as the knee extensors. It should also be kept in mind that the strength of the operator might be key in stabilizing the HHD device during the measurement procedure, and therefore the operator's own strength is likely to be of great importance for the accuracy of the measurements ${ }^{36)}$. 
When compared with previous results reporting the coefficient of variation $(\mathrm{CV})$ of isokinetic measurements using a ConTrex device (1.9-3.4\% for knee extensors and 2.7-3.6\% for knee flexors), the CVs reported in the present study are very similar for HHD (3.2-3.3\% for knee extensors and 3.7-4.2\% for knee flexors). This result suggests that the reproducibility of the strength measurements using HHD is in close agreement with that observed with isokinetic testing ${ }^{23)}$.

In a previous literature review comparing HHD with isokinetic dynamometry, Stark et al. showed that correlation coefficients ranged between 0.43 and 0.99 for the knee extensor and flexor muscles ${ }^{29}$ ). However, the heterogeneity of the protocols as well as the different models of dynamometers make the results difficult to interpret. The present study is the first to analyze the correlation between these specific dynamometers (ConTrex isokinetic vs MicroFet 2), and we report a good correlations between the 2 methods ( $r=0.72-0.87$ ). Of note, the present data were gathered using a simple protocol, easily reproducible without any additional equipment, in order to be established in routine clinical practice and to take the greatest advantage of the practical aspects of HHD.

When looking at subjects engaged in regular sports practice, only one study previously explored the correlation between HHD and isokinetic measurements ${ }^{37}$. The study reported lower correlation coefficients $(\mathrm{r}=0.334 \pm 0.11-0.617 \pm 0.7)$ compared to our results, but it should be emphasized that the number of subjects, the type of dynamometers, and the protocol employed were different.

Lastly, the present study is also the first to provide a correlation analysis between HHD and isokinetic testing for the evaluation of knee flexor/extensor muscle ratios, an index used routinely in the field of sports medicine. Our results show correlation coefficients ranging from 0.37 to 0.46 (all $\mathrm{p}<0.05$ ). Although statistically significant, these results indicate a moderate level of correlation between the 2 methodologies, and even no correlation at all when comparing flexor/extensor ratios obtained using HHD with that from isokinetic testing in eccentric mode. Therefore, flexor/extensor muscle strength ratios measured using HHD should be interpreted with caution until further investigation is performed in this area.

A limitation of our study is the fact that our population consisted of healthy, relatively young volunteers without osteoarticular or neuromuscular pathologies. Deones et al. compared muscle strength measurements by using hand-held and isokinetic dynamometers in a population of individuals with various unilateral orthopedic pathologies of the knee ${ }^{5)}$. They found a significant correlation between both methods ( $\mathrm{r}$ ranging from 0.57 to 0.80 ). However, the evaluation by HHD did not highlight a statistically significant difference between sides, whereas the isokinetic evaluation found a force deficit on the injured side. The pain that the patient may experience during the evaluation could be a confusing factor and must be taken into account when interpreting the strength results in the presence of pathologies. Another limit of this work is that HHD and isokinetic devices do not provide strength assessment with similar units of measurement. This makes direct comparison of absolute strength values difficult and limits the present comparison to correlation analysis. To obtain strength values expressed in the same units between the 2 devices (Newtons or kgf) would have required an evaluation of the lever arm, which is likely to have introduced more variability in the data.

Each evaluation method has its specific advantages and limitations. For a practitioner looking for rapid and regular muscle strength testing, the HHD allows rapid and easy acquisition of objective strength measurements. Knee extensor and flexor muscle strength recorded with an HHD is reproducible and significantly correlated with the isokinetic values, indicating that this method may in some cases be a useful replacement for isokinetic strength measurement, even for the assessment of strong muscles. However, for strength ratio assessment, and when judged against the isokinetic standard, HHD is not a valid option.

\section{REFERENCES}

1) Caron MA, Debigaré R, Dekhuijzen PN, et al.: [Diaphragm and skeletal muscle dysfunction in COPD]. Rev Mal Respir, 2011, 28: 1250-1264. [Medline] [CrossRef]

2) Middlekauff HR: Making the case for skeletal myopathy as the major limitation of exercise capacity in heart failure. Circ Heart Fail, 2010, 3: 537-546. [Medline] [CrossRef]

3) Campistol JM: Uremic myopathy. Kidney Int, 2002, 62: 1901-1913. [Medline] [CrossRef]

4) Walsmith J, Roubenoff R: Cachexia in rheumatoid arthritis. Int J Cardiol, 2002, 85: 89-99. [Medline] [CrossRef]

5) Deones VL, Wiley SC, Worrell T: Assessment of quadriceps muscle performance by a hand-held dynamometer and an isokinetic dynamometer. J Orthop Sports Phys Ther, 1994, 20: 296-301. [Medline] [CrossRef]

6) Reinking MF, Bockrath-Pugliese K, Worrell T, et al.: Assessment of quadriceps muscle performance by hand-held, isometric, and isokinetic dynamometry in patients with knee dysfunction. J Orthop Sports Phys Ther, 1996, 24: 154-159. [Medline] [CrossRef]

7) Malafarina V, Uriz-Otano F, Iniesta R, et al.: Sarcopenia in the elderly: diagnosis, physiopathology and treatment. Maturitas, 2012, 71: 109-114. [Medline] [CrossRef]

8) Kamiya K, Mezzani A, Hotta K, et al.: Quadriceps isometric strength as a predictor of exercise capacity in coronary artery disease patients. Eur J Prev Cardiol, 2014, 21: 1285-1291. [Medline] [CrossRef]

9) Alfano LN, Lowes LP, Flanigan KM, et al.: Correlation of knee strength to functional outcomes in Becker muscular dystrophy. Muscle Nerve, 2013, 47: 550-554. [Medline] [CrossRef] 
10) Suzuki M, Kirimoto H, Inamura A, et al.: The relationship between knee extension strength and lower extremity functions in nursing home residents with dementia. Disabil Rehabil, 2012, 34: 202-209. [Medline] [CrossRef]

11) Matsuzawa R, Matsunaga A, Wang G, et al.: Relationship between lower extremity muscle strength and all-cause mortality in Japanese patients undergoing dialysis. Phys Ther, 2014, 94: 947-956. [Medline] [CrossRef]

12) Hooten WM, Rosenberg CJ, Eldrige JS, et al.: Knee extensor strength is associated with pressure pain thresholds in adults with fibromyalgia. PLoS ONE, 2013, 8: e59930. [Medline] [CrossRef]

13) Yeung SS, Suen AM, Yeung EW: A prospective cohort study of hamstring injuries in competitive sprinters: preseason muscle imbalance as a possible risk factor. Br J Sports Med, 2009, 43: 589-594. [Medline] [CrossRef]

14) Lehance C, Binet J, Bury T, et al.: Muscular strength, functional performances and injury risk in professional and junior elite soccer players. Scand J Med Sci Sports, 2009, 19: 243-251. [Medline] [CrossRef]

15) Croisier JL, Ganteaume S, Binet J, et al.: Strength imbalances and prevention of hamstring injury in professional soccer players: a prospective study. Am J Sports Med, 2008, 36: 1469-1475. [Medline] [CrossRef]

16) Rochcongar P: [Isokinetic thigh muscle strength in sports: a review]. Ann Readapt Med Phys, 2004, 47: 274-281. [Medline] [CrossRef]

17) Croisier JL: Factors associated with recurrent hamstring injuries. Sports Med, 2004, 34: 681-695. [Medline] [CrossRef]

18) Croisier JL, Forthomme B, Namurois MH, et al.: Hamstring muscle strain recurrence and strength performance disorders. Am J Sports Med, 2002, 30: 199-203. [Medline]

19) Calmels P, Minaire P: A review of the role of the agonist/antagonist muscle pairs ratio in rehabilitation. Disabil Rehabil, 1995, 17: 265-276. [Medline] [CrossRef]

20) Hislop HJ, Perrine JJ: The isokinetic concept of exercise. Phys Ther, 1967, 47: 114-117. [Medline]

21) Li RC, Wu Y, Maffulli N, et al.: Eccentric and concentric isokinetic knee flexion and extension: a reliability study using the Cybex 6000 dynamometer. Br J Sports Med, 1996, 30: 156-160. [Medline] [CrossRef]

22) Croisier JL, Malnati M, Reichard LB, et al.: Quadriceps and hamstring isokinetic strength and electromyographic activity measured at different ranges of motion: a reproducibility study. J Electromyogr Kinesiol, 2007, 17: 484-492. [Medline] [CrossRef]

23) Maffiuletti NA, Bizzini M, Desbrosses K, et al.: Reliability of knee extension and flexion measurements using the Con-Trex isokinetic dynamometer. Clin Physiol Funct Imaging, 2007, 27: 346-353. [Medline] [CrossRef]

24) Almosnino S, Stevenson JM, Bardana DD, et al.: Reproducibility of isokinetic knee eccentric and concentric strength indices in asymptomatic young adults. Phys Ther Sport, 2012, 13: 156-162. [Medline] [CrossRef]

25) de Carvalho Froufe Andrade AC, Caserotti P, de Carvalho CM, et al.: Reliability of concentric, eccentric and isometric knee extension and flexion when using the REV9000 isokinetic dynamometer. J Hum Kinet, 2013, 37: 47-53. [Medline]

26) Kelln BM, McKeon PO, Gontkof LM, et al.: Hand-held dynamometry: reliability of lower extremity muscle testing in healthy, physically active,young adults. J Sport Rehabil, 2008, 17: 160-170. [Medline]

27) Hayes KW, Falconer J: Reliability of hand-held dynamometry and its relationship with manual muscle testing in patients with osteoarthritis in the knee. J Orthop Sports Phys Ther, 1992, 16: 145-149. [Medline] [CrossRef]

28) Toonstra J, Mattacola CG: Test-retest reliability and validity of isometric knee-flexion and -extension measurement using 3 methods of assessing muscle strength. J Sport Rehabil, 2013, Technical Notes. [Medline]

29) Stark T, Walker B, Phillips JK, et al.: Hand-held dynamometry correlation with the gold standard isokinetic dynamometry: a systematic review. PM R, 2011, 3: 472-479. [Medline] [CrossRef]

30) Stokes M: Reliability and repeatability of methods for measuring muscle in physiotherapy. Physiother Pract, 1985, 1: 71-76. [CrossRef]

31) Neder JA, Nery LE, Shinzato GT, et al.: Reference values for concentric knee isokinetic strength and power in nonathletic men and women from 20 to 80 years old. J Orthop Sports Phys Ther, 1999, 29: 116-126. [Medline] [CrossRef]

32) Bohannon RW: Reference values for extremity muscle strength obtained by hand-held dynamometry from adults aged 20 to 79 years. Arch Phys Med Rehabil, 1997, 78: 26-32. [Medline] [CrossRef]

33) Suzuki T: Reliability of measurements of knee extensor muscle strength using a pull-type hand-held dynamometer. J Phys Ther Sci, 2015, 27: 967-971. [Medline] [CrossRef]

34) Douma RK, Soer R, Krijnen WP, et al.: Reference values for isometric muscle force among workers for the Netherlands: a comparison of reference values. BMC Sports Sci Med Rehabil, 2014, 6: 10. [Medline] [CrossRef]

35) Lu YM, Lin JH, Hsiao SF, et al.: The relative and absolute reliability of leg muscle strength testing by a handheld dynamometer. J Strength Cond Res, 2011, 25: 1065-1071. [Medline] [CrossRef]

36) Wikholm JB, Bohannon RW: Hand-held dynamometer measurements: tester strength makes a difference. J Orthop Sports Phys Ther, 1991, 13: 191-198. [Medline] [CrossRef]

37) Whiteley R, Jacobsen P, Prior S, et al.: Correlation of isokinetic and novel hand-held dynamometry measures of knee flexion and extension strength testing. J Sci Med Sport, 2012, 15: 444-450. [Medline] [CrossRef] 